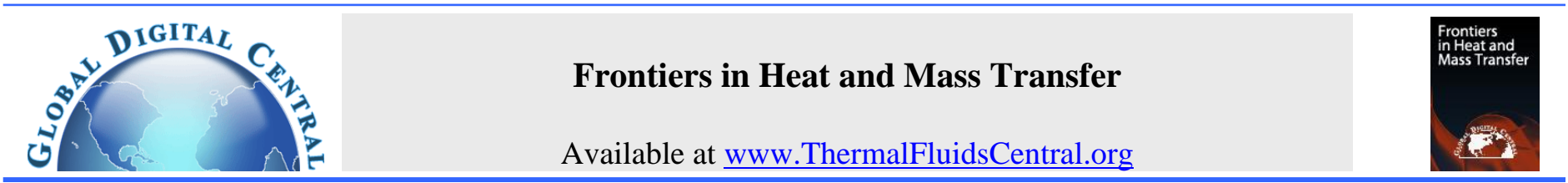

\title{
THERMAL ANALYSIS OF NATURAL CONVECTION AND RADIATION HEAT TRANSFER IN MOVING POROUS FINS
}

\author{
Partner L. Ndlovu ${ }^{\mathrm{a}, \mathrm{b}, *}$ and Raseelo J. Moitsheki ${ }^{\mathrm{a}, \dagger}$ \\ ${ }^{a}$ School of Computer Science and Applied Mathematics, University of the Witwatersrand, Johannesburg, Private Bag 3, Wits 2050, South Africa \\ ${ }^{\mathrm{b}}$ Standard Bank of South Africa, 30 Baker Street, Rosebank, Johannesburg, 2196, South Africa.
}

\begin{abstract}
In this article, the Differential Transform Method (DTM) is used to perform thermal analysis of natural convective and radiative heat transfer in moving porous fins of rectangular and exponential profiles. This study is performed using Darcy's model to formulate the governing heat transfer equations. The effects of porosity parameter, irregular profile and other thermo-physical parameters, such as Peclet number and the radiation parameter are also analyzed. The results show that the fin rapidly dissipates heat to the surrounding temperature with an increase in the values of the porosity parameter and the dimensionless time parameter. The results also show that the heat transfer rate in an exponential profile with negative power factor is much higher than the rectangular profile.
\end{abstract}

Keywords: Analytical Solutions, DTM, Darcy's model, heat transfer, fins

\section{INTRODUCTION}

Fins are extensively used in many heat transfer applications to enhance heat dissipation from a heated surface. An increased rate of heat transfer with reduced size and cost of fins is the main target for a number of engineering applications such as heat exchangers, conventional furnaces and gas turbines. Some engineering applications such as hydroplane and motorcycle also require a lighter fin with a higher rate of heat transfer. Increasing the heat transfer mainly depends on the heat transfer coefficient, the surface area available and the temperature difference between the surface and surrounding fluid. Extensive studies have been conducted to find an optimum shape and size for the fins to increase the heat transfer rate in different applications. The use of porous fins as a passive method for heat transfer enhancement has attracted a lot of research interests following the pioneer work of Kiwan and Al-Nimr (2001). Also, the research interests have aroused following the further studies by Kiwan (2007); Kiwani and Zeitoun (2008) on the thermal performance analysis of porous fins in natural convection environment. Following the work of Kiwan, most researchers have introduced other thermo-physical parameters to further improve the heat transfer from fins. Recently, a vast amount of research studies have been carried out by various authors in porous medium to improve the heat transfer process through fins.

The heat transfer rate by using porous medium can be further enhanced by increasing the effective area for the heat transfer by convection. The steady state thermal analysis of natural convection and radiation in porous fins was analytically conducted using Homotopy Analysis Method (HAM) by Moradi et al. (2014). These authors analyzed both infinite and finite fins with rectangular profile. Jooma and Harley (2017) studied a porous model for the radial fin. Contrary to their findings, the one dimensional DTM yielded approximate solutions which converge after thirteen iterations without requiring extra memory for construction of steady state solutions. Razelos and Kakatsios (2000) investigated the optimum dimensions of convecting-radiating rectangular fins. They studied the influence of all dependent parameters on optimization and performance of rectangular fins. They also investigated the effect of the temperaturedependent thermal conductivity and emissivity on the temperature profile.

In recent years, the heat transfer from a moving fin has attracted the attention of various researchers. This phenomenon is significant in numerous industrial applications such as extrusion, hot rolling, glass fiber drawing and casting. Turkyilmazoglu (2018) studied heat transfer from moving exponential fins with internal heat generation. Exact formulas for the thermal features like the distribution of temperature as well as the efficiency were derived and analyzed. The heat transfer of moving fins through a non-Newtonian fluid was first analytically studied by Fox et al. (1969). The effect of temperature-dependent thermal conductivity of the moving fin and radiation effect have been studied by Aziz and Khani (2011). They applied the HAM to solve governing equations and compared the analytical and numerical results. An analysis of heat transfer in a porous fin with temperature-dependent thermal conductivity and internal heat generation was conducted using Chebychev Spectral Collocation Method (CSCM) by Sobamowo (2017). This author used numerical solutions to investigate the effects of various parameters on the thermal performance of the porous fin. The results showed that increasing the porosity parameter also increases the rate of heat transfer from the base of the fin and consequently improve the efficiency of the fin. Colloca-

\footnotetext{
${ }^{\dagger}$ School of Computer Science and Applied Mathematics, University of the Witwatersrand, Johannesburg, Private Bag 3, Wits 2050, South Africa

${ }^{*}$ Corresponding author. Email: LuyandaNdlovu@icloud.com
} 
tion Method (CM) and the Homotopy Perturbation Method (HPM) were presented by Hoshyar et al. (2015) to determine the temperature distribution in a porous fin with temperature-dependent internal heat generation. Their results were validated by using the Runge-Kutta fourth order method. They discovered that as the buoyancy effects become stronger, the local temperature in the fin decreases. Following similar work undertaken by Hoshyar et al. (2015), Oguntala and Abd-Alhameed (2017) applied the Haar Wavelet Collocation Method (HWCM) to analyze the thermal performance of porous fins. A simulation of combined conductive, convective and radiative heat transfer in moving irregular porous fins of various profiles was performed by Ma et al. (2017) using Spectral Element Method (SEM). Besides, the effects of porous materials, profiles and various thermo-physical parameters on non-dimensional, the authors also investigated the temperature and volume adjusted fin efficiency. Gorla and Bakier (2011) solved a second order non-linear ordinary differential equation (ODE) describing heat transfer in a stationary porous fin using the Runge-Kutta fourth order method. It is found that in their analysis that radiates and convects transfers more heat than that dissipates heat by convection only. Kundu and Lee (2016) developed new analytical analysis by using double DTM to determine the temperature distribution in annular step porous fins subject to a mobile condition. They concluded that the porous fins have an ability to transfer more heat compared to the solid fin for an equal mass of fins at an optimum condition.

In this study, the DTM is applied to solve nonlinear heat transfer equation of a moving porous fins of rectangular and exponential profiles to determine temperature distribution along the fin. The effect of radiation on the performance of porous fin is studied in natural convection environment. We introduce a time factor to the governing equations resulting in highly nonlinear second order partial differential equations (PDEs). This improvement has not been developed in the related literature. The effect of porosity and radiation parameter, temperature ratio and Peclet number on dimensionless temperature and heat transfer rate is studied. The DTM is the most dominant method of analyzing temperature distribution through fin equations as it difficult to construct exact solutions. The DT$\mathrm{M}$ is an analytical method based on Taylor series expansion and was first introduced by Zhou (1986) in 1986. Zhou used the DTM to solve explicitly the linear and nonlinear initial value problems that arise in electrical circuits. Chen and Ho (1999), developed a two-dimensional DTM which can be used for solving differential and integral equations. Ayaz (2000), showed that the DTM is better equipped to solve highly nonlinear problems than the Taylor series method. The DTM has been used to solve various problems in applied mathematics and physics such as systems of differential equations (Kanth and Aruna, 2008).

This article is organized as follows; the mathematical background of the problem under consideration is described in $\S 2$. A brief discussion on the fundamentals of the 2D DTM will be provided in $\S 3$. We provide analytical solutions in $\S 4$. Lastly, we provide some discussions based on the results obtained in $\S 5$ and concluding remarks in $\S 6$.

\section{MATHEMATICAL FORMULATION}

We consider a moving porous fin of length $L$ and cross-sectional area $A_{c}$ while it moves horizontally with a constant velocity $U$ along an axial coordinate $X$ as depicted in Fig. 1. The fin surface is exposed to an environment of temperature $T_{a}$ and the base temperature of the fin is $T_{b}>T_{a}$. As the fin is porous, it allows fluid to penetrate through it, which enhances the convective heat transfer, The fin thickness at the prime surface is given by $\delta_{b}$ and its profile is given by $F(X)$. The following assumptions are considered for the problem under consideration:

- The fin tip is adiabatic.

- The temperature inside the fin is only function of $t$ and $X$.

- The physical properties of both solid wall and fluid are constant.
- The porous medium is homogeneous, isotropic and saturated with a single-phase fluid.

- The fluid and porous medium are not in local thermodynamic equilibrium.

- For the interaction between the porous medium and fluid, the Darcy's model is applied

The energy balance to the slice segment of the fin thickness $\Delta x$, as depicted in Fig. 1, requires:

$$
\begin{array}{r}
I=q(X)-q(X+\Delta X)-\dot{m} c\left(T-T_{a}\right) \\
\quad-P \Delta X \varepsilon \sigma\left(T^{4}-T_{a}^{4}\right)-\rho c A_{c} U \Delta X \frac{d T}{d X}
\end{array}
$$

where $\rho$ is the density of fluid; $c$ is the specific heat of fluid; $P$ is the periphery of the fin; $\varepsilon$ and $\sigma$ are the emissivity and Boltzman constant respectively; $T$ the temperature of the fin. $q$ is the heat flux of conduction. The time rate of change of internal energy in the volume element $I$ is given by,

$$
I=\rho c A_{c} \frac{d T}{d t} \Delta X
$$

By Fourier's law of conduction,

$$
q=-k_{c} A_{c} \frac{d T}{d X}
$$

where $k_{c}$ is the thermal conductivity of the fin. The mass flow rate of fluid $\dot{m}$ passing through the porous material is expressed by (Kiwan, 2007):

$$
\dot{m}=\rho \nu_{w} W \Delta X
$$

where $W$ is the width of the fin. The value of $\nu_{w}$ should be estimated from the consideration of the flow through the porous medium. Darcy's model then yields:

$$
\nu_{w}=\frac{g K \beta}{v}\left(T(X)-T_{a}\right) .
$$

Darcy's model stimulates the fluid-solid interaction in the porous medium. Here, $g$ is the gravitational acceleration, $K$ is the permeability, $v$ and $\beta$ are the kinematic viscosity and thermal expansion coefficient respectively. Substituting Equations (2) - (5) into Equation (1), the following nonlinear partial differential equation governing the temperature distribution in the fin is obtained:

$$
\begin{aligned}
\rho c \frac{\partial T}{\partial t} & =\frac{\delta_{b}}{2} \frac{\partial}{\partial X}\left(k_{c} F(X) \frac{\partial T}{\partial X}\right)-\frac{\rho c g K \beta W}{v A_{c}}\left(T-T_{a}\right)^{2} \\
& -\frac{P \sigma \varepsilon}{A_{c}}\left(T^{4}-T_{a}^{4}\right)-\frac{\delta_{b}}{2} F(X) \rho c U \frac{\partial T}{\partial X}, 0 \leq X \leq L,
\end{aligned}
$$

where $t$ is the time. $\delta_{b} F(X) / 2$ is introduced to the energy balance equation to cater for varying fin profiles. Assuming that the fin tip is adiabatic (insulated) and the base temperature is kept constant, then the boundary conditions are given by (Kraus et al., 2001; Kern and Kraus, 1972),

$$
T(t, L)=T_{b} \text { and }\left.\frac{\partial T}{\partial X}\right|_{X=0}=0,
$$

and initially the fin is kept at the ambient temperature (Ndlovu and Moitsheki, 2013),

$$
T(0, X)=T_{a} .
$$

Introducing the following dimensionless variables,

$$
\begin{aligned}
\theta & =\frac{T-T_{a}}{T_{b}-T_{a}}, x=\frac{X}{L}, \tau=\frac{k_{c} t}{\rho c L^{2}}, f(x)=\frac{\delta_{b}}{2} F(X), \\
\alpha & =\frac{k_{c}}{\rho c}, P e=\frac{U L}{\alpha}, N_{p}=\frac{g K \beta W L^{2}}{v \alpha A_{c}}\left(T_{b}-T_{a}\right), \\
N_{r} & =\frac{P \varepsilon \sigma L^{2}}{k_{c} A_{c}}\left(T_{b}-T_{a}\right)^{3} \text { and } N_{T}=\frac{T_{a}}{T_{b}-T_{a}}
\end{aligned}
$$


reduces Eqn. (6) to

$$
\begin{aligned}
\frac{\partial \theta}{\partial \tau} & =\frac{\partial}{\partial x}\left(f(x) \frac{\partial \theta}{\partial x}\right)-N_{p} \theta^{2}-N_{r}\left(\left(\theta+N_{T}\right)^{4}-N_{T}^{4}\right) \\
& -f(x) P e \frac{\partial \theta}{\partial x}, 0 \leq x \leq 1,
\end{aligned}
$$

and Eqn. (10) admits the following boundary conditions,

$$
\begin{gathered}
\theta(\tau, 1)=1, \tau>1, \\
\left.\frac{\partial \theta}{\partial x}\right|_{x=0}=0,
\end{gathered}
$$

and the initial condition becomes,

$$
\theta(0, x)=0
$$



Fig. 1. Schematic representation of a longitudinal porous fin of an unspecified profile.

Here, $N_{r}$ is the radiation parameter; $N_{T}$ is the dimensionless ratio of the surrounding temperature $T_{a}$ to the difference of the base temperature $T_{b}$ and surrounding temperature; $N_{p}$ is the porosity parameter that represents the effect of permeability of the porous medium beside the natural convection effects. The dimensionless variable $P e$ is the Peclet number which represent the dimensionless speed of the moving fin and $\mathrm{Pe}=0$ represents a stationary fin; $\theta$ is the dimensionless temperature, $x$ is the dimensionless space variable, $\tau$ is the non dimensional time.

\section{FUNDAMENTALS OF THE TWO-DIMENSIONAL DIFFERENTIAL TRANSFORM METHOD}

In this section, the basic idea underlying the two-dimensional DTM is briefly introduced. If function $\theta(t, x)$ is analytic and differentiated continuously with respect to time and the spatial variable $x$ in the domain of interest, then we let

$$
\Phi(\kappa, s)=\frac{1}{\kappa ! s !}\left[\frac{\partial^{\kappa+s} \phi(t, x)}{\partial t^{\kappa} \partial x^{s}}\right]_{(0,0)},
$$

where the spectrum $\Phi(\kappa, s)$ is the transformed function, which is also called the T-function (see Kangalgil and Ayaz (2009); Ayaz (2000)). The differential inverse transform of $\Phi(\kappa, s)$ is defined as

$$
\phi(t, x)=\sum_{\kappa=0}^{\infty} \sum_{s=0}^{\infty} \Phi(\kappa, s) t^{\kappa} x^{s}
$$

and from Eqns. (14) and (15) it can be concluded that

$$
\phi(t, x)=\sum_{\kappa=0}^{\infty} \sum_{s=0}^{\infty} \frac{1}{\kappa ! s !}\left[\frac{\partial^{\kappa+s} \phi(t, x)}{\partial t^{\kappa} \partial x^{s}}\right]_{(0,0)} t^{\kappa} x^{s}
$$

In real applications, the function $\phi(t, x)$ is expressed by a finite series, and Eqn. (15) can be written as:

$$
\phi(t, x)=\sum_{\kappa=0}^{m} \sum_{s=0}^{n} \Phi(\kappa, s) t^{\kappa} x^{s}
$$

Equation (17) implies that

$$
\phi(t, x)=\sum_{\kappa=m+1}^{\infty} \sum_{s=n+1}^{\infty} \Phi(\kappa, s) t^{\kappa} x^{s},
$$

is negligibly small.

Some of the useful mathematical operations performed by the differential transform method are given in Table 1 .

Table 1: Fundamental operations of the differential transform method

\begin{tabular}{ll}
\hline Original function & Transformed function \\
\hline$\phi(t, x)=f_{1}(t, x) \pm f_{1}(t, x)$ & $\Phi(\kappa, s)=F_{1}(\kappa, s) \pm F_{2}(\kappa, s)$ \\
$\phi(t, x)=\alpha f(t, x)$ & $\Phi(k, s)=\alpha F(\kappa, s)$ \\
$\phi(t, x)=\frac{\partial f(t, x)}{\partial t}$ & $\Phi(\kappa, s)=(\kappa+1) F(\kappa+1, s)$ \\
$\phi(t, x)=\frac{\partial f(t, x)}{\partial x}$ & $\Phi(\kappa, s)=(s+1) F(\kappa, s+1)$ \\
$\phi(t, x)=\frac{\partial^{2} f(t, x)}{\partial t^{2}}$ & $\Phi(\kappa, s)=(\kappa+1)(\kappa+2) F(\kappa+2, s)$ \\
$\phi(t, x)=\frac{\partial^{2} f(t, x)}{\partial x^{2}}$ & $\Phi(\kappa, s)=(s+1)(s+2) F(\kappa, s+2)$ \\
$\phi(t, x)=t^{m^{n}} x^{n}$ & $\Phi(\kappa, s)=\delta(\kappa-m) \delta(s-n)$ \\
$\phi(t, x)=x^{m} \exp (a t)$ & $\Phi(\kappa, s)=\frac{a^{\kappa}}{\kappa !} \delta(s-m)$ \\
\hline
\end{tabular}

The Kronecker delta function $\delta(\kappa-s)$ is given by

$$
\delta(\kappa-s)= \begin{cases}1 & \text { if } \kappa=s \\ 0 & \text { if } \kappa \neq s\end{cases}
$$

\section{DERIVATION OF ANALYTICAL SOLUTIONS}

In this section we provide analytical solutions for the problem described in $\S 2$. Using 2D-DTM to solve PDEs consists of three main steps. The steps are: transforming the PDE into algebraic equations, solving the equations, and inverting the solution of algebraic equations to obtain an infinite series solution or an approximate solution. The fin profile is rectangular when $f(x)=1$ and exponential when $f(x)=\mathrm{e}^{\eta x}$ where $\eta$ is a constant. Taking the two-dimensional differential transform of Eqn. (10) 
given a rectangular profile, we obtain the following recurrence relations,

$$
\begin{aligned}
& (\kappa+1) \Theta(\kappa+1, h)=(h+1)(h+2) \Theta(\kappa, h+2) \\
& -N_{p} \sum_{i=0}^{\kappa} \sum_{j=0}^{h} \Theta(i, h-j) \Theta(\kappa-i, j) \\
& -4 N_{r} N_{T}^{3} \Theta(k, h)-6 N_{r} N_{T}^{2} \sum_{i=0}^{\kappa} \sum_{j=0}^{h} \Theta(i, h-j) \Theta(\kappa-i, j) \\
& -4 N_{r} N_{T}^{2} \beta \sum_{i=0}^{\kappa} \sum_{l=0}^{\kappa-i} \sum_{j=0}^{h} \sum_{p=0}^{h-j} \Theta(i, h-j-p) \Theta(l, j) \Theta(\kappa-i-l, p) \\
& -N_{r} \sum_{i=0}^{\kappa} \sum_{l=0}^{\kappa-i} \sum_{z=0}^{\kappa-i-l} \sum_{j=0}^{h} \sum_{p=0}^{h-j} \sum_{t=0}^{h-j-p} \\
& \Theta(i, h-j-p-t) \Theta(l, j) \Theta(z, p) \Theta(\kappa-i-l-z, t) \\
& -\operatorname{Pe}(h+1) \Theta(k, h+1)
\end{aligned}
$$

where $\Theta(\kappa, h)$ is the differential transform of $\theta(\tau, x)$.

Taking the two-dimensional differential transform of the initial condition (13) and boundary condition (12) we obtain the following transformations respectively,

$$
\begin{gathered}
\Theta(0, h)=0, h=0,1,2, \ldots \\
\Theta(\kappa, 1)=0, \kappa=0,1,2, \ldots .
\end{gathered}
$$

We consider the other boundary condition as follows,

$$
\Theta(\kappa, 0)=a, a \in \mathbb{R}, \kappa=1,2,3, \ldots
$$

where the constant $a$ can be determined from the boundary condition (11) at each time step after obtaining the series solution.

Substituting Eqns. (20)-(22) into (19) we obtain the following,

$$
\begin{gathered}
\Theta(1,2)=c+2 c N_{r} N_{T}^{3} \\
\Theta(2,2)=\frac{1}{2}\left(3 c+c^{2} N_{p}+6 c^{2} N_{r} N_{T}^{2}+4 c N_{r} N_{T}^{3}\right) \\
\Theta(1,3)=\frac{1}{3}\left(P e\left(c+2 c N_{r} N_{T}^{3}\right)\right) \\
\Theta(2,3)=\frac{1}{6}\left(P e\left(3 c+c^{2} N_{p}+6 c^{2} N_{r} N_{T}^{2}+4 c N_{r} N_{T}^{3}\right)\right)
\end{gathered}
$$

Substituting Eqns. (20)-(26) into (17) we obtain the following closed form of the solution,

$$
\begin{aligned}
\theta(\tau, x) & =a \tau+a \tau^{2}+a+2 a N_{r} N_{T}^{3} \tau x^{2} \\
& +\frac{1}{2}\left(3 a+a^{2} N_{p}+6 a^{2} N_{r} N_{T}^{2}+4 a N_{r} N_{T}^{3}\right) \tau^{2} x^{2} \\
& +a \tau^{3}+\frac{1}{3}\left(\operatorname{Pe}\left(a+2 a N_{r} N_{T}^{3}\right)\right) \tau x^{3} \\
& +\frac{1}{6}\left(\operatorname{Pe}\left(3 a+a^{2} N_{p}+6 a^{2} N_{r} N_{T}^{2}+4 a N_{r} N_{T}^{3}\right)\right) \tau^{2} x^{3} \\
& +\ldots
\end{aligned}
$$

The constant $a$ can be determined from the boundary condition (11) at each time step. To obtain the value of $a$, we substitute the boundary condition (11) into (27) at the point $x=1$. Thus, we have,

$$
\begin{aligned}
\theta(\tau, 1) & =a \tau+a \tau^{2}+\left(a+2 a N_{r} N_{T}^{3}\right) \tau \\
& +\frac{1}{2}\left(3 a+a^{2} N_{p}+6 a^{2} N_{r} N_{T}^{2}+4 a N_{r} N_{T}^{3}\right) \tau^{2} \\
& +a \tau^{3}+\frac{1}{3}\left(\operatorname{Pe}\left(a+2 a N_{r} N_{T}^{3}\right)\right) \tau \\
& +\frac{1}{6}\left(\operatorname{Pe}\left(3 a+a^{2} N_{p}+6 a^{2} N_{r} N_{T}^{2}+4 a N_{r} N_{T}^{3}\right)\right) \tau^{2} \\
& +\ldots=1
\end{aligned}
$$

We then obtain the expression for $\theta(\tau, x)$ upon substituting the obtained value of $a$ into equation (27). Following a similar approach, we obtain the following solution for the exponential fin profile,

$$
\begin{aligned}
\theta(\tau, x) & =a \tau+a \tau^{2}+\left(a+2 a N_{r} N_{T}^{3}\right) \tau x^{2} \\
& +\frac{1}{2}\left(3 a+a^{2} N_{p}+6 a^{2} N_{r} N_{T}^{2}+4 a N_{r} N_{T}^{3}\right) \tau^{2} x^{2} \\
& +a \tau^{3}+\frac{1}{3}\left(a P e+2 a P e N_{r} N_{T}^{3}-2 a \eta-4 a N_{r} N_{T}^{3} \eta\right) \tau x^{3} \\
& +\left(2 a+a^{2} N_{p}+6 a^{2} N_{r} N_{T}^{2}+2 a^{3} N_{r} N_{T}^{2}+2 a N_{r} N_{T}^{3}\right) \tau^{3} x^{2} \\
& +\ldots
\end{aligned}
$$

Using the first 20 terms of the power series solution, we benchmark the series solutions to the numerical solution obtained through employing the Maple package. The pdsolve in Maple uses Finite Difference Method (FDM) to discretize the differential equation. The technique is a centered implicit scheme that is capable of finding solutions to higher-order partial differential equations. As seen from the benchmark analysis in Fig. 2, the series solution is in agreement with the numerical solution across all profiles.

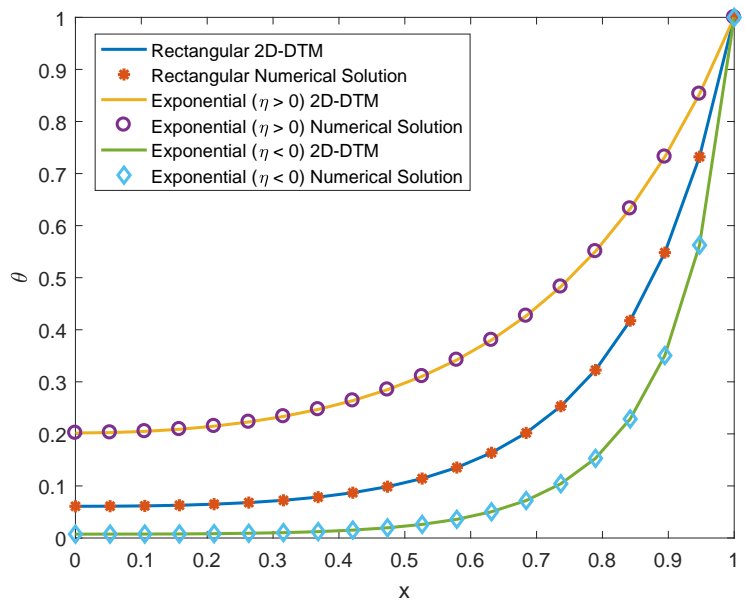

Fig. 2. Temperature distribution in differing fin profiles.

With the confidence obtained from the benchmark analysis, we plot the solutions for (27) and (29) for various parameters as shown in Figs. below. For all analytical results reported in this article, the following values of parameters are used unless stated otherwise as indicated in the graphs below.

$$
N_{p}=10, N_{r}=5, N_{T}=0.10, P e=3, \tau=0.6
$$

\section{SOME DISCUSSIONS}

In this article, we have established analytical expressions for determination of thermal performance in moving porous fins with three different profiles. Figs. 3 and 4 shows that as time evolves, the fin temperature increases across different profiles as a result of thermal conduction. Fig. 5 depicts the variation of temperature distribution with varying values of $N_{T}$. We note that the temperature on the fin decreases with the increasing values of $N_{T}$. This signifies that the heat transfer rate reaches its peak with the increasing values of $N_{T}$ while other parameters are kept constant. The only way to increase $N_{T}$ is through changing the value of the ambient temperature for a fixed base temperature. The effect of the porosity parameter $N_{p}$ is shown in Fig. 6. Expectedly, as the values of the porosity parameter increase, the heat transfer rate to the ambient fluid is increased as evidenced by the rapid decrease of the dimensionless 


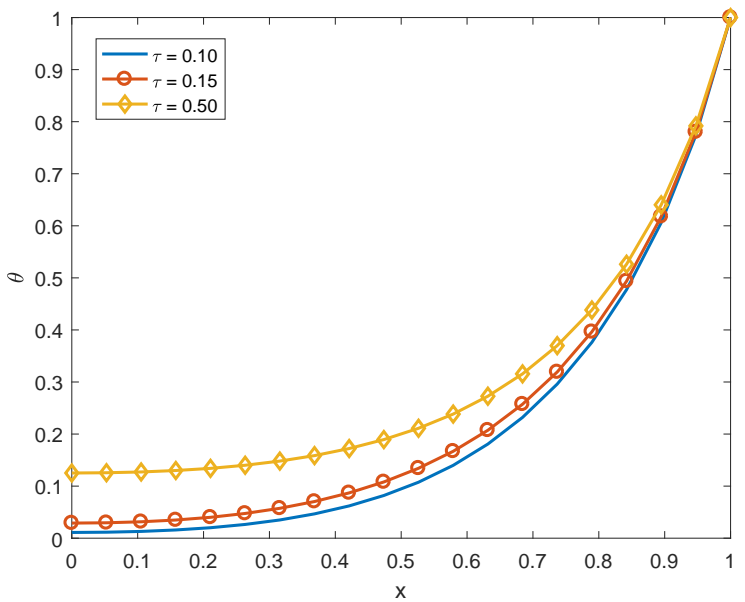

Fig. 3. Temperature distribution in a longitudinal rectangular fin for varying time, $\tau$.

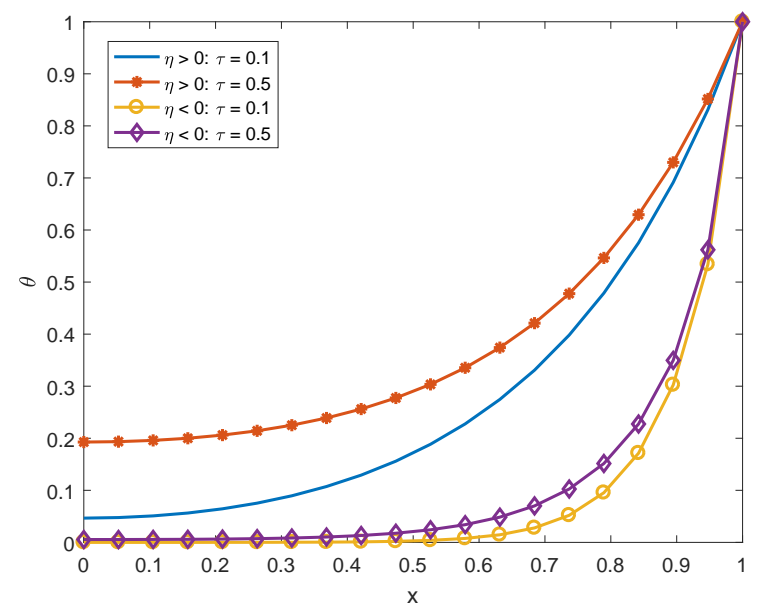

Fig. 4. Temperature distribution in a longitudinal exponential fin for varying time, $\tau$.

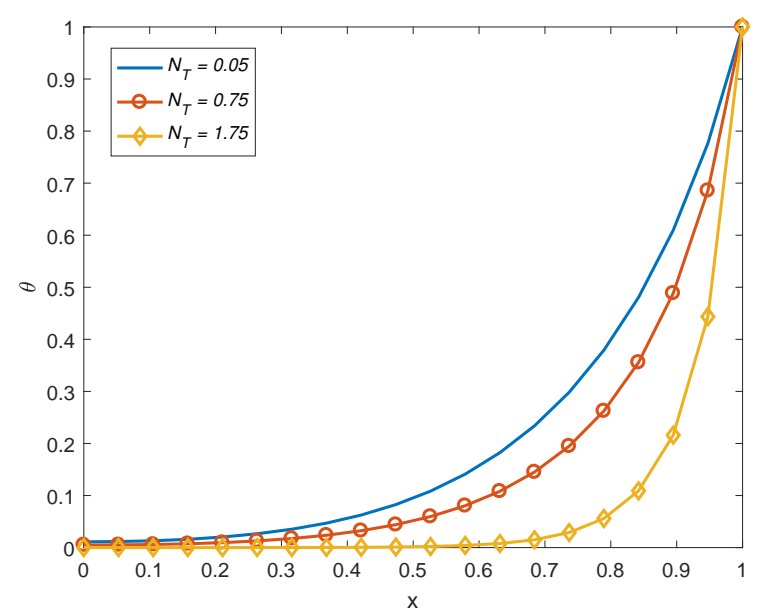

Fig. 5. Effect of the dimensionless ratio of temperature, $N_{T}$, on a rectangular fin temperature distribution.

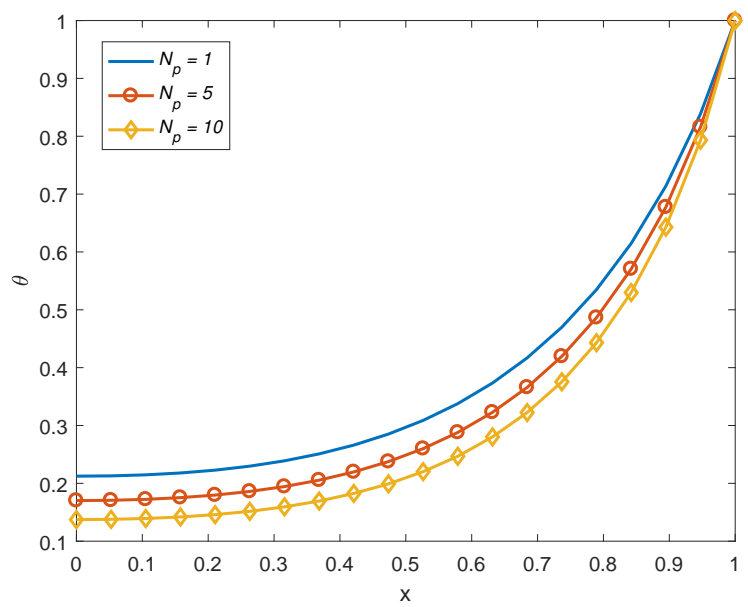

Fig. 6. Effect of the porosity parameter, $N_{p}$, on a rectangular fin temperature distribution.

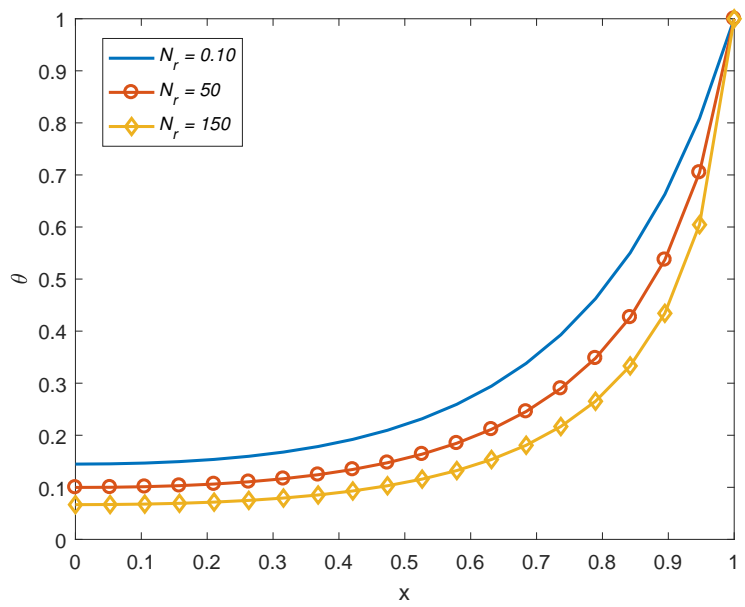

Fig. 7. Effect of the radiation parameter, $N_{r}$, on a rectangular fin temperature distribution.

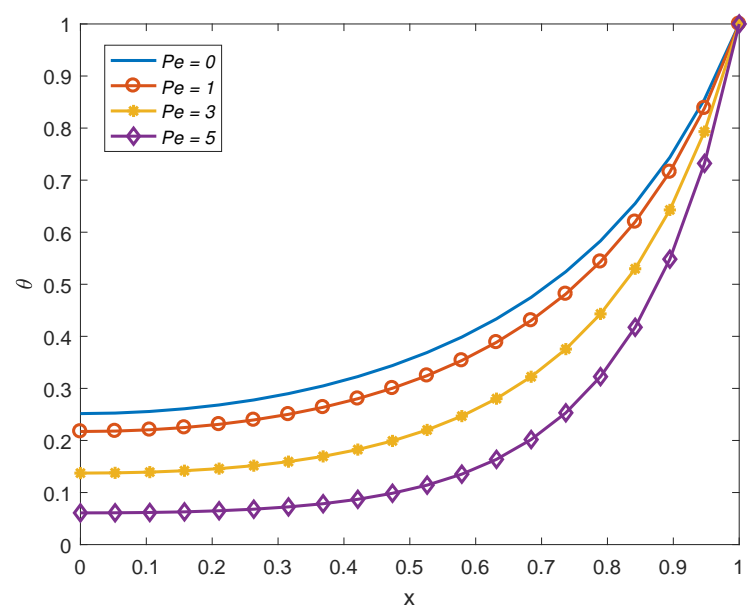

Fig. 8. Effect of varying speeds, $P e$, on a rectangular fin temperature distribution. 




Fig. 9. Temperature distribution for differing axial values in a rectangular fin.

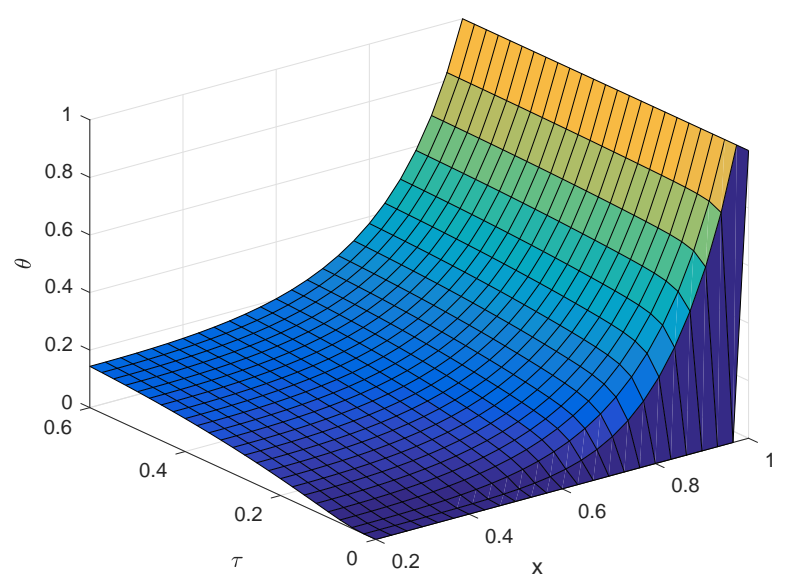

Fig. 10. Transient temperature distribution in a longitudinal rectangular fin.

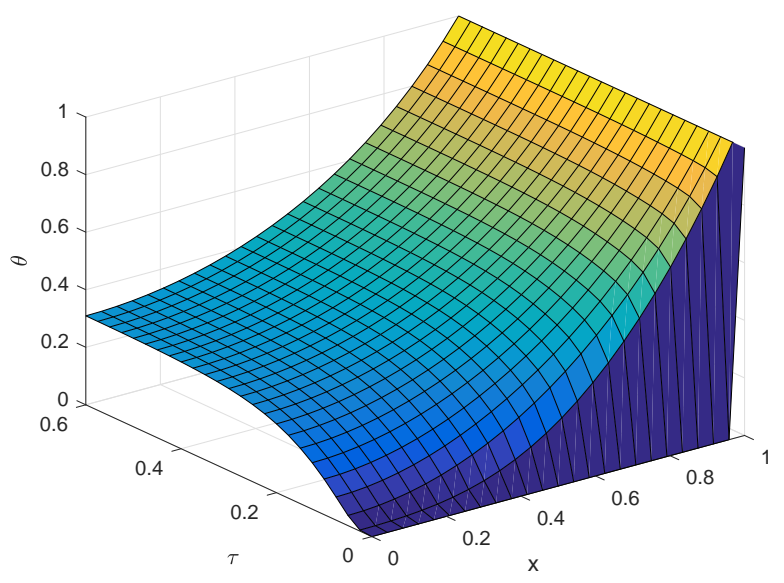

Fig. 11. Transient temperature distribution in a longitudinal exponential fin, $\eta=2$.

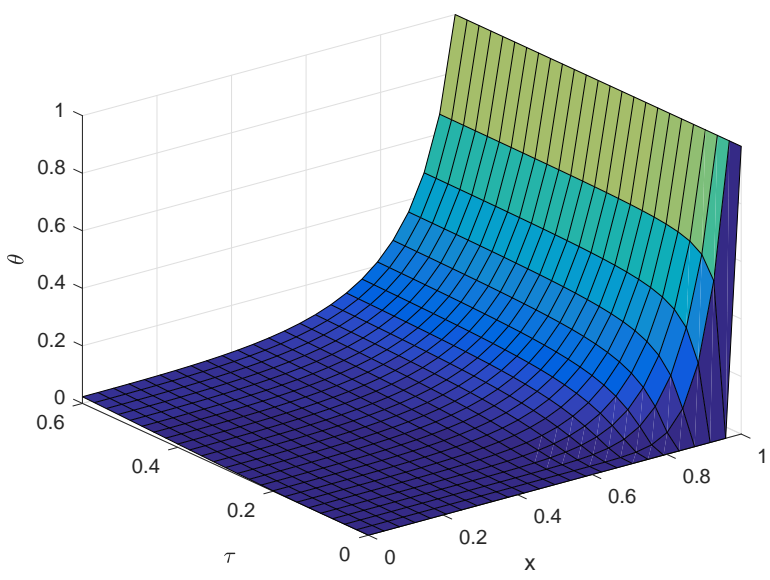

Fig. 12. Transient temperature distribution in a longitudinal exponential fin, $\eta=-2$.

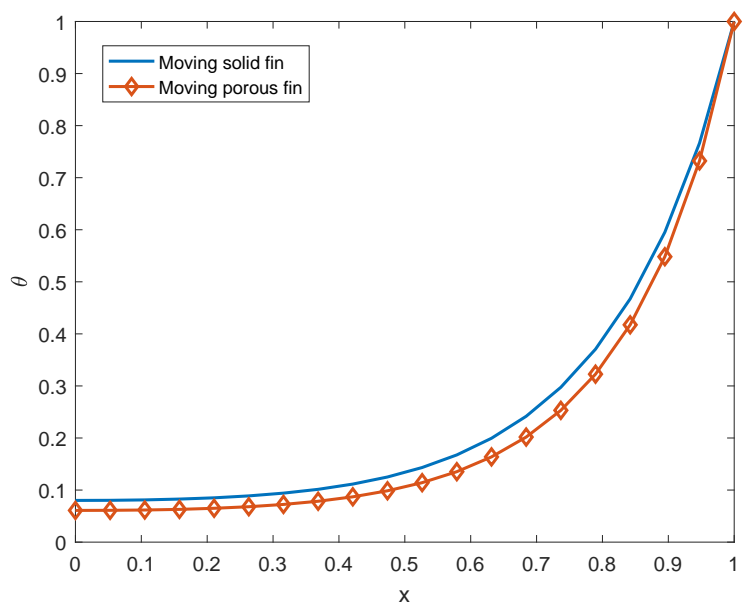

Fig. 13. Effect of different porous media in a longitudinal rectangular fin.

temperature inside the fin. In the case of a porous fin exposed to natural convection, higher porosity due to changing of permeability included in $N_{p}$ results in higher rate of heat transfer. Physically, this can be caused by the effect of larger area of porous medium. Fig. 7 shows the effect of the radiation parameter $N_{r}$ on temperature distribution. We note that as radiation gets stronger, the fin loses heat to the ambient fluid effectively as shown by the decreasing temperatures inside the fin. It can be deduced from the results that thermal energy transfers by radiation enhance heat transfer rate from fin. The intensity of such energy flux depends upon the temperature difference between fin and ambient and the nature of fin's surface.

The impact of the speed of the moving fin as defined by the Peclet number $P e$ is shown in Fig. 8. We also observe that for $P e=0$, i.e., stationery fin, the cooling of the fin takes more time as shown by higher temperatures when compared to a moving fin. Physically, this is the ratio of the rate of advection of thermal energy by the flow to the rate of thermal energy diffusion driven by an appropriate gradient. As $P e$ increases, that is, the fin moves quicker, the advection effect becomes stronger and the fin dissipates heat rapidly as indicated by the lower temperatures on inside the fin. Consequently, where cooling is required, the higher value of Peclet number is desirable. Fig. 9 shows the variation in temperature distribution for different values of the axial parameter $x$, that is, the temperature variation along the fin profile. We see that as the the axial 
variable increases, i.e, as we approach the fin base, the temperature inside the fin increases. The comparison between the fin of rectangular profile and that of an exponential profile is show in Fig. 2. We see that the fin of exponential profile with a negative power is more efficient in dissipating heat to the surrounding fluid. The validation of the results and the boundary conditions are shown as three dimensional plots in Figs. 10, 11 and 12. Lastly, Fig. 13 shows that the moving porous fin dissipates heat faster and efficiently to the the surrounding fluid that the moving solid fin.

\section{CONCLUDING REMARKS}

In this study, we have illustrated that the temperature distribution in the moving porous fin is highly dependent upon the fin profile. This is an important finding for fin design as we noted that an exponential porous fin is more efficient than the solid fin. We also discovered that the temperature distribution in a moving porous fin can be affected by changes in the underlying physical parameters. The DTM was used to perform thermal analysis as it is the most dominant analytical method for these kinds of problems and has been found to converge faster to the exact solutions with a appropriate number of iterations considered.

\section{ACKNOWLEDGEMENTS}

RJM thanks the National Research Foundation South Africa for financial support.

\section{NOMENCLATURE}

c specific heat $(\mathrm{J} / \mathrm{kg} \cdot \mathrm{K})$

$h \quad$ heat transfer coefficient $\left(\mathrm{W} / \mathrm{m}^{2} \cdot \mathrm{K}\right)$

$k \quad$ thermal conductivity $(\mathrm{W} / \mathrm{m} \cdot \mathrm{K})$

$k_{c} \quad$ thermal conductivity $(\mathrm{W} / \mathrm{m} \cdot \mathrm{K})$

$t \quad$ time (s)

$T \quad$ temperature (K)

$U \quad$ velocity of the fin $(\mathrm{m} / \mathrm{s})$

$X \quad$ space variable $(\mathrm{m})$

$x \quad$ dimensionless space variable $(\mathrm{m})$

$A_{c} \quad$ cross-sectional area $\left(\mathrm{m}^{2}\right)$

$L \quad$ fin length (m)

$L \quad$ fin profile

$W \quad$ fin width

$T_{a} \quad$ ambient temperature (K)

$T_{b} \quad$ fin base temperature (K)

$P \quad$ fin perimeter

$K \quad$ permeability of the porous medium

$P e \quad$ Peclet number

$\Phi(t, x) \quad$ transformed analytical function

$\phi(t, x) \quad$ original analytical function

$q \quad$ heat flux $\left(\mathrm{W} / \mathrm{m}^{2}\right)$

$\dot{m} \quad$ mass flow rate of fluid $(\mathrm{Kg} / \mathrm{s})$

$N_{r} \quad$ radiation parameter

$N_{T} \quad$ temperature ratio

$N_{p} \quad$ porosity parameter

$g \quad$ gravitational acceleration $\left(\mathrm{m} / \mathrm{s}^{2}\right)$

Greek Symbols

$\sigma \quad$ Boltzmann constant $\left(\mathrm{W} / \mathrm{m}^{2} \cdot \mathrm{K}^{4}\right)$

$\delta_{b} \quad$ base fin width (m)

$\varepsilon \quad$ total emissivity

$\beta \quad$ thermal expansion coefficient $\left(\mathrm{K}^{-1}\right)$

$\rho \quad$ density $\left(\mathrm{kg} / \mathrm{m}^{3}\right)$

$\tau \quad$ dimensionless temperature

$\rho \quad$ density $\left(\mathrm{kg} / \mathrm{m}^{3}\right)$

$\alpha \quad$ thermal diffusivity $\left(\mathrm{m}^{2} / \mathrm{s}\right)$

$v \quad$ kinematic viscosity $\left(\mathrm{m}^{2} / \mathrm{s}\right)$

$\theta \quad$ dimensionless temperature

\section{REFERENCES}

Al-Nimr, M.A., Kiwan, S., 2001. "Using Porous Fins for Heat Transfer Enhancement", Journal of Heat Transfer, 123(4), 790-795. http://dx.doi.org/10.1115/1.1371922

Kiwan, S., 2007. "Effect of radiative losses on the heat transfer from porous fins", International Journal of Thermal Sciences, 46, 1046-1055. https://doi.org/10.1016/j.ijthermalsci.2006.11.013

Kiwan, S., Zeitoun, O., 2008. "Natural convection in a horizontal cylindrical annulus using porous fins", International Journal of Numerical Heat and Fluid Flow, 18(5), 618-634.

https://doi.org/10.1108/09615530810879747

Moradi, A., Fallah, A.P.M., Hayat, T., Aldossary, O. M., 2014. “On Solution of Natural Convection and Radiation Heat Transfer Problem in a Moving Porous Fin", Arabian Journal for Science and Engineering, 39, 1303-1312.

http://dx.doi.org/10.1007/s13369-013-0708-9

Jooma, R., Harley, C., 2017. "Heat transfer in a porous radial fin: Analysis of numerically obtained solutions", Advances in Mathematical Physic$s$, Article ID 1658305

https://doi.org/10.1155/2017/1658305

Razelos, P., Kakatsios, X., 2000. "Optimum dimensions of convectingradiating fins: Part I - longitudinal fins", Applied Thermal Engineering, 20, 1161-1192.

https://doi.org/10.1016/S1359-4311(99)00089-7

Turkyilmazoglu, M., 2018. "Heat transfer from moving exponential fins exposed to heat generation", International Journal of Heat and Mass Transfer, 116, 346-351.

https://doi.org/10.1016/j.ijheatmasstransfer.2017.08.091

Fox, V.G., Erickson, L.E., Fan, L.T., 1969. “The laminar boundary layer on a moving continuous flat sheet immersed in a non-Newtonian fluid", AIChE. Journal, 15(3), 327-333.

https://doi.org/10.1002/aic.690150307

Aziz, A., Khani, F., 2011. "Convection-radiation from a continuously moving fin of a variable thermal conductivity", Journal of Franklin Institute, 348, 640-651.

https://doi.org/10.1016/j.jfranklin.2011.01.008

Sobamowo, M.G., 2017. "Analysis of Heat transfer in Porous Fin with Temperature-dependent Thermal Conductivity and Internal Heat Generation using Chebychev Spectral Collocation Method", Journal of Computational and Applied Mechanics, 48(2), 271-284.

http://dx.doi.org/10.22059/jcamech.2017.239131.171

Hoshyar, H.A., Rahimipetroudi, I., Ganji, D.D., Majidian, A.R., 2015. "Thermal performance of porous fins with temperature-dependent heat generation via the homotopy perturbation method and collocation method", Journal of Applied Mathematics and Computational Mechanics, 14(4), 53-65.

http://dx.doi.org/10.17512/jamcm.2015.4.06

Oguntala, G., Abd-Alhameed, R., 2017. "Haar Wavelet Collocation Method for Thermal Analysis of Porous Fin with Temperature-dependent Thermal Conductivity and Internal Heat Generation", Journal of Applied and Computational Mechanics, 3(3), 185-191.

http://dx.doi.org/10.22055/JACM.2017.21494.1103

Ma, J., Sun, Y., Li, B., 2017. "Simulation of combined conductive, convective and radiative heat transfer in moving irregular porous fins by spectral element method", International Journal of Thermal Sciences, 118, 475-487.

https://doi.org/10.1016/j.ijthermalsci.2017.05.008 
Gorla, R.S.R., Bakier, A.Y., 2011. "Thermal analysis of natural convection and radiation in porous fins", International Communications in Heat and Mass Transfer, 38, 638-645.

https://doi.org/10.1016/j.icheatmasstransfer.2010.12.024

Kundu, B., Lee, K., 2016. "A proper analytical analysis of annular step porous fins for determining maximum heat transfer", Energy Conversion and Management, 110, 469-480.

https://doi.org/10.1016/j.enconman.2015.09.037

Zhou, J.K., 1986. "Differential Transform Method and Its Applications for Electric Circuits", Huazhong University Press, Wuhan, China.

Chen, C.K., Ho, S.H., 1999. "Solving partial differential equations by two-dimensional differential transform method", Applied Mathematics and Computation, 106, 171-179.

https://doi.org/10.1016/S0096-3003(98)10115-7

Ayaz, F., 2003. "On the two-dimensional differential transform method", Applied Mathematics and Computation, 143, 361-374. https://doi.org/10.1016/S0096-3003(02)00368-5

Ravi Kanth, A.S.V., Aruna, K., 2008. "Differential transform method for solving linear and nonlinear systems of partial differential equations", Physics Letters, 372, 6896-6898.

http://dx.doi.org/10.1016/j.physleta.2008.10.008

Kraus, A.D., Aziz, A., Welty, J., 2001. "Extended Surface Heat Transfer", Wiley, New York.
Kern, Q.D., Kraus, D.A., 1972. "Extended Surface Heat Transfer", McGraw-Hill, New York.

Moradi, A., Rafiee, R., 2013. "Analytical solution to convectiveÜradiative of continuously moving fin with temperature-dependent thermal conductivity", International Journal of Thermal Sciences, 17, 1049-1060. https://doi.org/10.2298/TSCI110425005M

Ndlovu, P.L., Moitsheki, R.J., 2013. "Application of the two-dimensional differential transform method to heat conduction problem for heat transfer in longitudinal rectangular and convex parabolic fins", Communications in Nonlinear Science and Numerical Simulation, 18, 2689-2698. https://doi.org/10.1016/j.cnsns.2013.02.019

Ndlovu, P.L., Moitsheki, R.J., 2013. "Conservation laws and associated Lie point symmetries admitted by the transient heat conduction problem for heat transfer in straight fins", Central European Journal of Physics, 11(8), 984-994.

https://doi.org/10.2478/s11534-013-0306-1

Kangalgil, F., Ayaz, F., 2009. "Solitary wave solutions for the KdV and mKdV equations by differential transform method", Chaos, Solitons and Fractals, 41, 464-472.

https://doi.org/10.1016/j.chaos.2008.02.009 